\title{
POLÍTICAS HABITACIONAIS: RESULTADOS E LACUNAS NA PRODUÇÃO LATINO-AMERICANA
}

\author{
HOUSING POLICIES: OUTCOMES AND GAPS FROM LATIN \\ AMERICAN ACADEMIC PRODUCTION \\ POLÍTICAS DE VIVIENDA: RESULTADOS Y BRECHAS EN LA \\ PRODUCCIÓN LATINOAMERICANA
}

\section{Paula Neumann Novack}

Bacharela e Mestra em Geografia pela Universidade Federal de Pelotas. Doutoranda em Geografia na Pontificia Universidad Católica de Chile. Bolsista da Comisión Nacional de Investigación Científica y Tecnológica de Chile (CONICYT). Av. Vicuña Machenna 4860, Macul/Santiago - Chile, Campus San Joaquín. E.mail: paulanovack@gmail.com

\section{Rodrigo Hidalgo}

Doutor em Geografia Humana pela Universidad de Barcelona. Professor no Instituto de Geografia da Pontificia Universidad Católica de Chile e Chefe do Programa de Doutorado em Geografia da mesma universidade. Av. Vicuña Machenna 4860, Macul/Santiago - Chile, Campus San Joaquín. E.mail: rodrigohidalgogeo@gmail.com

\section{RESUMO}

O artigo apresenta uma análise bibliográfica das políticas habitacionais e suas consequências nos espaços urbanosda América Latina. A metodologia utilizada foi a revisão dos resultados de sessenta artigos publicados em bases de dados e selecionados a partir das palavras-chave políticas habitacionais e urbano. Analisados a partir de como se associam a distintas escalas, os principais temas encontrados foram o direito à cidade, déficit habitacional, programas habitacionais (escala nacional), plano diretor, processo de segregação, infraestrutura urbana (escala cidade), autogestão, apropriação e assentamentos irregulares (escala bairro).Resultados indicam que as políticas habitacionais em áreas centrais e espaços ociosos da cidade são investigadas considerando o processo de gentrificação, mas poderiam ser estudadas a partir de outras implicações espaciais. Conclui-se apontandoa necessidade de avançar em pesquisas que priorizem a participação da população nos processos de discussão e planejamento de políticas habitacionais.

Palavras-chave: Políticas Habitacionais; Geografia Urbana;América Latina; Revisão de Literatura. 


\begin{abstract}
This work presents a literature analysis of housing policies and their consequences in Latin American urban space. The methodology used was a review of outcomes from more than sixty papers published and selected from databases using terms housing policies and urban. Analyzed from what scales are associated to them, main founded issues were right to the city, housing deficit, housing programs (national scale), city plan, segregation, infrastructure (city scale), self-management, appropriation and irregular settlements (neighborhood scale). Results outline that housing policies in central areas and empty spaces are researched considering gentrification process, otherwise could be analyzed from other spatial implications. Conclusion points out the need of move forward in investigation that prioritizes people's participation in housing policies discussions and planning.
\end{abstract}

Keywords: Housing Policies; Urban Geography; Latin America; Literature Review.

\title{
RESUMEN
}

El artículo presenta un análisis bibliográfico sobre las políticas de vivienda y sus consecuencias en los espacios urbanos de la América Latina. La metodología utilizada fue la revisión de los resultados de sesenta artículos publicados en bases de datos y seleccionados a partir de las palabras-clave políticas de vivienda y urbano. Analizados a partir de cómo se asocian las distintas escalas, los principales temas encontrados fueron el derecho a la ciudad, déficit habitacional, programas de vivienda (escala nacional), plan director, proceso de segregación, infraestructura urbana (escala ciudad), autogestión, apropiación, asentamientos irregulares (escala barrio). Resultados indican que las políticas de vivienda en áreas centrales y espacios ociosos de la ciudad son investigadas tomando en cuenta el proceso de gentrificación, pero podrían ser estudiados a partir de otras implicaciones espaciales. Se concluye apuntando la necesidad de avanzar en investigaciones que prioricen la participación de la población en los procesos de discusión y planificación de políticas de vivienda.

Palabras-clave: Políticas de Vivienda; Geografía Urbana; América Latina; Revisión de Literatura. 


\section{INTRODUÇÃO}

A habitação se mostra como uma discussão relevante no contexto das cidades latinoamericanas, este é um tema que se apresenta como um problema das cidades nos chamados países em desenvolvimento. O tema das políticas habitacionais precisa ser aprofundado para a solução de parte dos problemas urbanos e da população que vive nas cidades. Por isso, cabe identificar as temáticas relacionadas a estas políticase a partir disso, levantar questões que devem ser discutidas e aprofundadas para solucionar e melhorar as condições de acesso e de qualidade de vida nas cidades latino-americanas.

Como direito a habitação é reivindicada por diversos setores da sociedade. No espaço urbano ela acabou tornando-se um dos principais articuladores da expansão e, por consequência, da segregação. Diversos geógrafos vêm dedicando-se a entender como ser desdobram esses movimentos com distintas estratégias de análise. Dessa forma, se procura entender como as políticas habitacionais são observadas nas distintas escalas de produção do espaço urbano. A partir disso, se identificam as lacunas de análise e os temas que poderiam ser trabalhados considerando o contexto latino-americano. Para responder este objetivo, se realizou um estudo sobre as políticas habitacionais centrandose na produção em português e espanhol, em seguida se apresenta os principais eixos de análise sobre o tema e finalmente, se realiza apontamentos sobre as temáticas a serem desenvolvidas considerando as políticas habitacionais.

Os temas relacionados as políticas habitacionais se manifestam em distintas escalas de análise. Neste sentido, o artigo apresenta temas que se destacam em três escalas, a nível nacional comoo direito à cidade, o déficit habitacional, os programashabitacionais pensados de acordo com as necessidades de determinado país. Depois se trabalha com a escala a nível de cidade onde se destacam os temas de plano diretor que de forma geral busca regular as cidades, também as políticas habitacionais implantadas em diferentes contextos urbanos, o processo segregação e o tema da infraestrutura urbana que aparece em distintas perspectivas nos artigos que trabalham com políticas habitacionais. Por fim, se apresenta uma discussão das políticas habitacionais relacionadas com uma escala ainda menor, considerando o bairro. Nesse sentido se trabalha com os temas de autogestão/ autoconstrução, apropriação e também com assentamentos irregulares.

O artigo está estruturado em quatro partes, nesta primeira se apresenta o tema investigado com os objetivos e apontamentos iniciais. A segunda parte trabalha especificamente com a metodologia aplicada para esta análise, métodos de busca, palavras-chave, filtros aplicados e outros aspectos gerais. Na terceira parte são apresentados os resultados que são classificados 
por escalas de análise (Nacional, Cidade, Bairro). Finalmente, são apresentadas algumas considerações e apontamentos sobre os resultados e lacunas sobre o tema.

\section{METODOLOGIA}

Sobre os aspectos metodológicos aplicados para a realização do artigo, cabe destacar a busca sobre o tema das políticas habitacionais na base de dados SCOPUS e também em revistas brasileiras selecionadas a partir do sistema Qualis Capes (2014). Este sistema classifica as revistas com base em critérios de qualidade e relevância cientifica, neste sentido foram selecionadas as revistas com Qualis A1, A2, B1.

Primeiramente, se realizou uma busca na base de dados SCOPUS, onde inicialmente se encontrou 564 artigos que trabalham com o tema. A partir disso, se utilizaram alguns filtros para investigação conforme quadro 1 .

\section{Quadro 1: Critérios de busca}

1. Palavras-chave: housing polic*, urban

2. Período: 2006 a 2016

3. Tipo de documento: Artigos

4. Idiomas: Português, Espanhol

5. Área: Ciências Sociais

Fonte: Elaborado pela Autora (Maio/2016)

Com a filtragem foi possível realizar uma análise prévia dos resultados quantitativos, onde se destaca que da pesquisa inicial restaram 50 artigos, depois de uma análise qualitativa, seis artigos não atendiam ao foco da pesquisa e foram descartados, resultando em um total de 44 artigos para a análise referente a base SCOPUS. Com respeito às revistas brasileiras, se utilizou o sistema Qualis Capes (QC) de avaliação de periódicos para selecionar os artigos. Os filtros utilizados para a busca em revistas brasileiras foram os mesmos aplicados para a base de dados SCOPUS. Como mencionado anteriormente, da lista de revistas avaliadas pelo sistema QC, foram utilizadas para a pesquisa aquelas que apresentaram QC A1, A2, B1.

Da busca realizada em revistas brasileiras, foram selecionados 16 artigos que trabalham com as políticas habitacionais relacionadas com a produção do espaço urbano no Brasil. A pesquisa em revistas brasileiras se justifica porque o país tem uma significativa contribuição sobre o tema, considerando principalmente os altos investimentos empolíticas habitacionais nos últimos anos, onde é possível destacar a relevância do Programa Minha Casa Minha Vida. Além disso, a maioria das suas revistas e periódicos não estão vinculadas a base de dados como SCOPUS, Web of Science e outros. Por isso, se realizou uma busca especifica 
no sistema Qualis Capes, conformando um total de 60 artigos para amostra desta análise bibliográfica.

A categoria empregada para análise dos resultados é a escala geográfica, entendendose essa pelo espaço atribuído a um evento social. Parte-se da ideia Marston(2000)da simultaneidade com que a escala se manifesta no local. Portanto, é o propósito desse trabalho separá-la metodologicamente e analisar como os distintos níveis interatuam na produção do local. Três níveis foram determinados para distribuir os temas associados à habitação: 1) uma escala nacional, mediadora das políticas públicas e macro discursos acerca da habitação; 2) uma escala cidade, apontando as gerenciais especificas de cada realidade urbana e 3) uma escala de bairro, buscando experienciais e especificidades dos projetos de habitação e dos sujeitos que neles atuam.

\section{Detalhes da amostra}

Com relação a base de dados SCOPUS, a pesquisa identificou que as revistas com mais publicações sobre o tema são a Revista del Instituto de la Vivienda (INVI)e a Revista Eure. Com respeito aos países mais investigados se destacam Chile com dezesseis publicações, Brasil com nove e Argentina com sete.Ainda sobre a pesquisa realizada em esta base de dados, se destaca que as cidades mais investigadas são Santiago do Chile com nove publicações, Córdoba na Argentina com quatro, Buenos Aires com duas, Valparaíso com também com duas e somam mais quatro publicações os trabalhos que pesquisam mais de uma cidade.

Sobre os dezesseis artigos selecionados através da análise de revistas brasileiras se destaca que seis pesquisas trabalham sobre estudos de caso no Estado de São Paulo, nas cidades de São Paulo e Campinas. Além disso, dois artigos realizam uma comparação entre as políticas habitacionais no Brasil com outros países, entre eles estão Chile, Argentina e Paraguai. Outros dois artigos trabalham com as políticas habitacionais em escala nacional, e ainda aparecem artigos que trabalham especificamente com a cidade de Fortaleza, Aracaju, Porto Alegre, Curitiba e Uberlândia.As palavras mais frequentes nos artigos são políticas habitacionais, produção do espaço, habitação de interesse social e plano diretor.

\section{RESULTADOS E DISCUSSÃO}

Para organização dos dados foi utilizada uma metodologia de sistematização com base no conceito de escala. Através da leitura dos artigos se identificou que os mesmos seguiam uma escala nacional, de cidade ou de bairro. A escala nacional apresenta alguns temas que ultrapassam a dimensão local e que estão relacionadas as questões habitacionais mais gerais que se repetem em distintos países na América Latina. A escala cidade discute outros 
pontos importantes que influenciam as políticas habitacionais considerando o espaço local onde alguns processos se repetem com algumas peculiaridades, considerando a dinâmica de cada cidade. Quando se trabalham os temas de Plano Diretor, processo de segregação e infraestrutura urbana, os artigos analisados sempre se referem a uma cidade específica onde estes fenômenos ocorrem.

Por fim, a escala de bairro apresenta temas que se destacam considerando uma dimensão local ainda menor e mais específica. Os temas aqui apresentados estão ligados ao espaço vivido diferentemente dos pontos trabalhados para a escala de cidade que estão voltados ao espaço concebido, onde se destacam temas relacionados com planejamento urbano e suas consequências.

\section{Quadro 2: Escalas de análise e suas principais temáticas}

\begin{tabular}{l|ll}
\multicolumn{1}{c|}{ NACIONAL } & \multicolumn{1}{c|}{ CIDADE } & \multicolumn{1}{c}{ BAIRRO } \\
\hline Direito à cidade / Direito à Habitação & Plano Diretor & Autogestão/Autoconstrução \\
\hline Déficit Habitacional & Processo de Segregação & Apropriação \\
\hline Programas Habitacionais & Infraestrutura Urbana & Assentamento Irregular \\
\hline
\end{tabular}

Fonte: Elaborado pela Autora (Maio/2016)

\section{Escala nacional}

Nesta análise de escala nacional se destacam os temas de direito à cidade vinculado ao direito à habitação, o déficit habitacional e aos programas habitacionais que são mais relevantes nas análises.

Esta escala apresenta também uma relação com o papel e a intervenção do Estado levando em consideração o tema das políticas habitacionais. Os três temas apresentados têm ligação com as inciativas do Estado frente as discussões e prioridades para um melhor desempenho dessas políticas. São temas gerais que podem ser pensados em distintas escalas de análise, mas que na análise dos artigos selecionados, se manifestam como uma discussão nacional.

\section{Direito à cidade/ direito à habitação}

Com respeito ao direito à cidade e à habitação Quintana (2014)afirma que no Chile, durante o governo de Eduardo Frei Montalva, a habitação é apresentada como um direito de toda família e que o Estado tem que auxiliar economicamente as pessoas que possuem poucos recursos.Este discurso de apoio a população também é evidente durante o governo de Salvador Allende, onde a habitação é vista como um direito de todos e o Estado deve 
proporciona-la priorizando a qualidade de vida das pessoas e não o lucro. Ribeiro \& Moreira (2014)destacam que o Estatuto da cidade instituiu o direito à cidade como princípio social fundamental, definindo instrumentos urbanísticos ara valorizar os direitos de participação da população em decisões sobre o processo de produção do espaço urbano como, por exemplo, a implementação de políticas de habitação. Nos artigos analisados, o tema do direito à cidade e à habitação se apresentam como algo muito importante para o desenvolvimento das cidades, mas isso não ocorre em casos concretos. $O$ direito à cidade se manifesta na maioria dos casos como uma teoria, uma utopia que não se realiza na prática.

\section{Déficit habitacional}

O déficit habitacional é um ponto que se apresenta frequentemente nos artigos analisados, este é um tema que impulsiona as iniciativas e as políticas de habitação de uma forma geral. Todas as políticas e programas de habitação surgem em um contexto de solução ao problema de déficit habitacional. Este sempre tem como objetivo a solução para os problemas urbanos como, por exemplo, a precariedade das habitações em que as pessoas vivem, relacionadas com ao restrito acesso aos recursos e equipamentos urbanos.

Monteiro (2015) utiliza o exemplo da cidade de Curitiba para falar das políticas que buscam a solução do problema do déficit habitacional, da precariedade urbana e das habitações no Brasil. Assim, o tema de déficit habitacional também está relacionado com o rápido crescimento da população urbana.Rubin (2014) em seu estudo sobre as políticas de habitação no Chile e no Brasil, destaca que os dois países tinham problemas para solucionar o déficit de habitação. Conclui que no Chile o desenvolvimento das políticas para solucionar esse problema se manifesta de forma mais eficaz, mas na verdade os dois países cometeram muitos erros no planejamento e execução dessas políticas.

Shimbo (2013)ressalta que o Brasil tem um grande problema de déficit habitacional. Em seu estudo, destaca que o aproveitamento das construções antigas ou abandonadas nos centros urbanos podem ser uma proposta relevante para ajudar a solucionar esse problema em grandes cidades. Cabe destacar que os números de déficit habitacional são compatíveis com o número de construções ociosas ou vazias. Além do caso brasileiro, com a análise de Quintana (2014), é possível realizar uma leitura das iniciativas que buscam através de políticas públicas e do direito à habitação, solucionar os problemas de déficit habitacional no Chile em diferentes épocas e governos. 


\section{Programas habitacionais}

Nos artigos analisados, o tema das políticas habitacionais está vinculado a uma dinâmica Nacional, depois de realizar um panorama das políticas nacionais, eles partem para uma análise de estudos de caso mais específicos. Os artigos brasileiros apresentam o tema das políticas públicas como uma solução para o déficit habitacional do país. Nesse sentido, o Programa Minha Casa Minha Vida (PMCMV) é fortemente citado nas análises, destacando distintas perspectivas.

O artigo de Rolnik, Iacovini \& Klintowitz (2014), analisa a participação popular no projeto de construção e implementação de empreendimentos imobiliários do PMCMV nos municípios da cidade de São Paulo. A pesquisa conclui que a opinião e a percepção das pessoas não são consideradas durante o processo de planejamento, construção e realização dos conjuntos habitacionais. O trabalho deKopper (2016), indica que o PMCMV é uma iniciativa do Estado para a solução dos problemas de déficit habitacional, é também uma grande oportunidade de fortalecimento e reprodução do mercado imobiliário no Brasil.

As pesquisas de Freitas, Renato \& Pequeno (2009) e Baltrusis \& Mourad (2014)destacam que na década de 2000 houve um aumento significativo dos recursos para a construção de habitações de baixa renda, possibilitando novos mercados habitacionais. Com tudo, o artigo de Freitas et al. (2009)questionam até que ponto essas medidas favoreceram o acesso da população de baixa renda aos espaços urbanizados. A conclusão é que muitos empreendimentos solucionam o problema da casa própria, no entanto, os problemas de acesso aos equipamentos urbanos permanecem.

Delgadillo (2008) apresenta uma análise sobre programas e projetos habitacionais voltados para a reabilitação urbana de áreas centrais em cidades latino-americanas. Em seu artigo é possível observar discussões relacionadas ao esvaziamento dos centros, deterioração de prédios históricos, também novos investimentos imobiliários que culminam em atividades terciarias. O autor ressalta a relevância desses projetos e programas voltados para as áreas centrais como uma possibilidade de resolver problemas relacionados ao déficit habitacional, promover a recuperação do patrimônio edificado e também como uma alternativa para melhorar os acessos da população aos equipamentos urbanos.

Realizando uma breve análise das políticas e programas de habitação presente nos artigos, é possível concluir que os problemas de habitação são parcialmente resolvidos com essas iniciativas. Isso ocorre por distintos motivos, as pessoas têm o problema da habitação solucionado, mas essa solução não se mostra suficiente para a realização de uma vida digna, porque para isso, é necessário muito mais que um teto. Por isso, os artigos que trabalham 
com as políticas de habitação destacam a importância da participação das pessoas no processo de planejamento dos programas, com foco social, não somente relacionado ao capital e ao mercado imobiliário.

\section{Escala cidade}

Os principais temas vinculados a cidade, tais temas foram agrupados em três itens principais, representados pelos planos diretores, o processo de segregação e também os temas relacionados a infraestrutura urbana.A escala cidade diferentemente da escala nacional apresenta temas mais específicos e que podem variar de acordo com as peculiaridades de cada cidade. Todos esses processos que são pensados a nível nacional se manifestam de distintas formas a nível de cidade,porque no espaço local operam diferentes lógicas que se desdobram em organizações urbanas distintas.

\section{Plano Diretor}

No caso dos planos diretores das cidades, é possível destacar que ele tem o papel de regular e apontar algumas diretrizes para uma melhoria nas questões que se relacionam com as políticas de habitação. Em muitos planos diretores, existe uma sessão para discutir e esclarecer os temas vinculados aos programas de habitação, melhoria dos bairros e habitações existentes.

O texto deOliveira Santos (2013), retrata a importância do plano diretor participativo para discussão conjunta com a política municipal habitacional de Fortaleza, com o objetivo de contribuir para as políticas urbanas de habitação. No entanto, se chega à conclusão que os planejamentos das cidades seguem uma lógica perversa, onde somente parte das pessoas são contempladas com as diretrizes dos planos diretores, porque seu planejamento não é estendido para toda a cidade. Com tudo, o autor destaca a relevância da existência do plano diretor e que também é importante buscar, pouco a pouco, melhorias com o máximo de participação da população que vive nas cidades.

Para Machado (2012) o plano diretor deve ser bem elaborado para que seja possível minimizar os problemas de qualidade de vida em ambientes urbanos, destaca que no caso da cidade de Aracajú o plano diretor possui grandes contradições porque ele mesmo contribui para a heterogeneidade econômica e também para os processos de segregação na cidade. $\mathrm{O}$ autor destaca que é importante discutir e escrever sobre as deficiências dos planos diretores e com isso, realizar uma proposta de reformulação positiva dos planos que conduzem as cidades. 


\section{Processo de segregação}

O processo segregação espacial está presente nas cidades contemporâneas, em grandes cidades e em regiões metropolitanas este processo é ainda mais intenso. Marengo (2013) tem como objetivo realizar um diagnóstico sobre a segregação residencial na cidade de Córdoba/Argentina para depois, orientar as novas políticas de habitação e apontar suas possíveis localizações. Assim, se realiza uma reflexão sobre as limitações do planejamento urbano e as políticas de habitação. A autora conclui que os planos de regulação têm muitos problemas e por isso, as brechas sociais são amplas, a cidade se expande e intensifica as condições de inequidade no acesso à habitação, aos equipamentos urbanos e coloca em questão a sustentabilidade dos modelos de crescimento urbano.

Em Oliveira \& Pedlowski (2012), o processo de segregação se apresenta em conjunto com as políticas de habitação implantadas em Campos dos Goytacazes/Brasil. O autor destaca que, em muitos casos, as políticas habitacionais são desenvolvidas em locais muito distantes dos centros das cidades, fortalecendo a segregação espacial nos espaços urbanos. No Brasil, estes casos são bem comuns, grandes áreas residenciais são construídas muito longe dos centros urbanos, criando verdadeiras ilhas sociais. Como consequência desse processo, as pessoas não têm a oportunidade de viver a cidade, ou seja, pelo emprego no mercado de trabalho formal ou pelo acesso aos serviços públicos essenciais. Neste contexto, os conjuntos habitacionaissão um fator de segregação, não de integração e democratização.

É possível dizer que o tema da segregação espacial está fortemente presente na discussão do espaço urbano e das cidades. Este fenômeno ocorre em distintas dinâmicas urbanas e é ainda mais visível em regiões metropolitanas como apresentam os trabalhos deHidalgo \& Zunino (2011)e Martori \& Hoberg (2006). A segregação na América Latina se justifica também porque as cidades cresceram rapidamente e não tinham estrutura para receber tantas pessoas em pouco tempo. Os artigos analisados destacam que é fundamental pensar a segregação como um fenômeno espacial que deve ser minimizado através de políticas públicas e políticas habitacionais. É fundamental criar possibilidade de inclusão de acesso aos serviços urbanos nos espaços segregados e não pensar em elimina-los do contexto da sociedade urbana.

\section{Infraestrutura urbana}

As cidades Latino-Americanas apresentam muitos problemas de infraestrutura, nesse sentido, é possível destacar o trabalho de Duarte (2012)que fala sobre os problemas ambientais que são criados a partir da construção de habitações irregulares em áreas que não estão preparadas para a construção, ou seja, que apresentam um problema de 
infraestrutura urbana. Isso ocorre porque as pessoas se instalam e constroem suas casas em lugares onde não existe nenhum tipo de investimento em infraestrutura urbana. $\mathrm{Na}$ sua pesquisa, o autor destaca um caso específico de habitações construídas em um lugar sem infraestrutura, com tudo, este lugar está em processo de recuperação por conta do dialogo participativo entre população e Estado.

No trabalho de Shimbo (2013) o problema de infraestrutura urbana se apresenta relacionado ao abandono das áreas centrais urbanas. Na sua pesquisa, a infraestrutura presente no centro da cidade de São Paulo se apresenta como um recurso para a implantação de projetos de habitação. Neste trabalho, a autora discute como a infraestrutura ociosa pode ser aproveitada pelos programas de habitação, através da construção de novas habitações.

Berr, Echeveste, Lorenzi \& Formoso (2015) destacam os problemas de qualidade das habitações sociais desde a percepção dos moradores. A conclusão é que as habitações têm problemas de infraestrutura e que os programas habitacionais devem investir um pouco mais na construção de qualidade. Finalmente, se pode dizer que o tema da infraestrutura urbana está presente em distintas perspectivas nas análises dos artigos. Na maioria dos casos, a infraestrutura é vista como deficiente e como um problema de que deve ser melhorado no contexto das políticas de habitação e no contexto do urbano. Também existem casos onde a infraestrutura não é vista como um problema e sim como algo que deve ser repensado e aproveitado para o desenvolvimento das cidades.

\section{Escala de bairro}

Para finalizar a análise dos artigos sobre políticas habitacionais, serão trabalhadas as temáticas que foram identificadas à nível de bairro. Os temas que serão aprofundados são a autoconstrução - autogestão, a discussão acerca da apropriação e a configuração dos assentamentos irregulares.Como mencionado anteriormente, nessa perspectiva se desdobram os temas relacionados ao espaço vivido que se manifestam em uma dimensão micro escalar. Neste contexto operam os atores locais que vivem e alteram a dinâmica do bairro com suas ações diárias, que ultrapassam a dimensão do espaço concebido e o planejamentodas cidades.

\section{Autoconstrução/ autogestão}

A autoconstrução e a autogestão são temas que se apresentam numa escala micro, nos bairros, assentamentos e comunidades específicas. Castillo (2014)trabalha com o papel do habitante na produção de seu habitat e em sua relação com a política pública de habitação e bairro no Chile. As conclusões de suas análises enfatizam que os pobres urbanos estão 
consolidando, com o tempo, ascompetências para construir habitação e melhorar seus bairros. Essas habilidades, lhes permitem participar do processo de programas de habitação de pequena escala, até a política habitacional de grande escala.

A autora trabalha, neste caso, com a Población Obrera de La Unión de Valparaíso e resume as aprendizagens adquiridas pela comunidade através do processo de autogestão, autoconstrução e gestão comunitária em três principais pontos. Entre eles, se destaca a organização para convivência, as práticas solidárias para a vida em comunidade e a resistência ao despejo. Assim, cabe ressaltar que a autogestão e a autoconstrução analisam as ações que as pessoas de determinado lugar empreendem para consolidar seu hábitat e para conseguir o acesso à terra, estas ações são relacionadas com as políticas públicas e programas de habitação.

Em outro artigo,Castillo \& Forray (2014) destacam que os altos preços das habitações são fatores que fortalecem o processo de autoconstrução. Existem muitos questionamentos sobre o termo habitação social porque, em muitos casos, se entende que parte das pessoas não podem pagar pela habitação e por isso, os processos de ocupação de terrenos e de autoconstrução ocorrem. Sobre a análise do tema da autoconstrução é possível concluir que este processo vai continuar acontecendo em quanto não houver acesso a habitação por um preço justo, de acordo com as condições financeiras da população.

\section{Apropriação}

A apropriação ocorre em todas as escalas analisadas. No entanto, este processo é mencionado com maior frequência em questões ligadas ao hábitat e a ocupação de terrenos para autoconstrução de habitações. Neste sentido, cabe destacar novamente os artigos de Castillo \& Forray (2014), que trabalham com o processo de apropriação de lugares para a autoconstrução de habitações. Também é possível citar o trabalho de Gonçalves (2010) que investiga o processo de apropriação em uma escala ainda menor, relacionado a casa. A autora destaca as transformações que ocorrem nas habitações desde o momento em que as pessoas passam a habita-las. A apropriação da casa transforma o hábitat em habitar, através deste processo são criados os símbolos, os significados na relação que se estabelece entre a casa e o morador.

Assim, é importante dizer que o processo de apropriação se apresenta como um fator que se manifesta em distintas dimensões, com tudo, ele se destaca na escala de bairro, relacionado aos temas de autoconstrução, ocupação de lugares e especialmente em estudos onde se apresenta a construção da relação entre os moradores e suas casas. 


\section{Assentamentos irregulares}

Os assentamentos irregulares são também relacionados ao processo de autoconstrução, ocupação de terras e a apropriação de terrenos, como citado anteriormente. Nesse sentido, se apresentam numa escala micro, considerando uma área limitada da cidade. A criação dos assentamentos está também vinculada ao processo de segregação, ou seja, funcionam como uma resposta das pessoas aos projetos de habitação que são construídos em espaços segregados da cidade.

Por isso, cabe citar o trabalho de Valenzuela, Suárez \& Downey (2010) que apresenta a discussão dos assentamentos irregulares na região metropolitana de Santiago. Esses assentamentos buscam desde sua criação, responder ao processo de implantação de políticas habitacionais que são construídas em áreas periféricas, fortalecendo o processo de segregação espacial e expulsão dos pobres da cidade. Assim, é importante assinalar que a construção de acampamentos está relacionada também com a decisão das pessoas em viver longe de todas as possibilidades de ter progresso pessoal e familiar. Esses acampamentos são também uma forma de chamar atenção das autoridades para o problema da habitação.

Neste caso citado por Valenzuela et al. (2010), é possível destacar a resistência das pessoas a formação do que chamamos de guetos e a formação de grandes zonas de homogeneidade social que as políticas habitacionais realizam nas periferias, longe da cidade. Outro fator que deve ser mencionado nas análises dos acampamentos, é a sua dinâmica. Os assentamentos irregulares são lugares que apresentam muitas mudanças na sua configuração, em respeito às famílias que fazem parte de sua estrutura, a todo momento, pessoas entram e saem em busca de melhores oportunidades de viver na cidade.

\section{CONSIDERAÇÕES FINAIS}

O artigo apresenta uma análise bibliográfica sobre as políticas habitacionais. O objetivo principal da análise é identificar os resultados e lacunas sobre o tema. Para alcançar este objetivo, primeiramente, se realizou uma análise detalhada sobre os principais temas e elementos trabalhados nos artigos selecionados. Se realizou também, uma análise das metodologias presentes nos artigos e a partir disso, foi possível identificar alguns resultados e lacunas para avançar e contribuir em estudos sobre as políticas habitacionais.

Como parte dos resultados e lacunas, é possível destacar a necessidade de trabalhar com as políticas habitacionais desenvolvidas em áreas centrais e espaços ociosos da cidade. A maior parte dos artigos encontrados a partir da palavra-chave políticas habitacionais, 
estão vinculados a discussões de condomínios, programas, projetos que são desenvolvidos em áreas distantes da cidade ligados aos temas de segregação, problemas de infraestrutura e planejamento. As pesquisas, de forma geral, não trabalham com a origem desses processos que ocorrem a partir da expulsão, da exclusão de pessoas das cidades e centros urbanos.

Sobre essa primeira lacuna apontada, é importante dizer que somente dois artigos apresentam uma discussão sobre as políticas habitacionais nos espaços ociosos da cidade como é o caso dos centros históricos de grandes cidades. Muitos são os casos de metrópoles que têm prédios de sua área central abandonados, de forma geral estes espaços são dirigidos para processos de reocupação, gentrificação. Por outro lado, são poucas investigações que trabalham como análise de políticas de habitação direcionadas para estes lugares. Por tanto, esta seria uma temática para novas investigações com respeito as políticas habitacionais.

Outra lacuna identificada é a falta de trabalhos sobre a percepção, experiência e apropriação das populações com respeito as políticas de habitação. Também se identifica a falta de trabalhos etnográficos e de cartografia social. Além disso, poucas pesquisas utilizam metodologias de observação participativa. Essas lacunas foram identificadas através das análises das metodologias dos artigos selecionados, uma delas está relacionada com o pequeno número de pesquisas que trabalham com a percepção das pessoas com respeito a habitação e os lugares onde vivem. Métodos etnográficos e observação participativa para avaliar as políticas habitacionais são pouco utilizados. Assim, se caracteriza mais um resultado com respeito a essa análise.

Além disso, também não existem referencias metodológicas que considerem a experiência das pessoas com relação a apropriação da casa, sua participação nos projetos de planejamento dos programas e das políticas habitacionais. Nesse sentido, é possível dizer que são poucas as fontes de análise que trabalham com materiais pessoais, familiares e de história oral como fonte de pesquisa. O uso da cartografia se manifesta muito limitado, em uma lógica quantitativa de avaliação de crescimento, localização. Em consequência, nenhum artigo apresenta o uso da cartografia social e métodos que produzam mapeamentos mais críticos e avançados com relação as políticas habitacionais.

Em suma, os resultados e lacunas identificados até agora, fazem referência a falta de pesquisas com uma análise mais crítica dos programas e das políticas habitacionais. $\mathrm{O}$ que se identifica, na maioria dos casos, é uma análise bibliográfica e documental sobre o tema. Existem também artigos que realizam uma mescla de metodologias quantitativas e qualitativas. No entanto, nestes casos, se observa que os resultados das pesquisas apresentam muito mais dados numéricos, que as análises qualitativas das entrevistas realizadas. 
Finalmente, cabe dizer que é necessário colocar ênfase qualitativa nas análises das políticas habitacionais porque as análises bibliográficas, históricas e quantitativas já expressam um número significativo.Estas lacunas mostram que com respeito aos aspectos metodológicos seria interessante avançar nas pesquisas que priorizam a participação efetiva das populações na discussão acerca das políticas habitacionais. Também se aponta como fundamental investigar as políticas habitacionais presentes em áreas centrais e espaços ociosos das cidades, com ênfase nasimplicações destas políticas, que se manifestam além do processo de gentrificação.

\section{REFERÊNCIAS}

6. BALTRUSIS, N.; MOURAD, L. N. Política habitacional e locação social em salvador. Caderno CRH, 2014. v. v. 27, n. n. 71, p. 267-284.

7. BERR, L. R. et al. Indicador de falhas de qualidade baseado na percepção dos usuários de habitação de interesse social. Ambiente construído, 2015. p. 19-35. Disponível em: <http:// www.scielo.br/scielo.php?script=sci_arttext\&pid=S1678-86212015000400019\&lang=pt $>$. CASTILLO, M. J. Pobladores: potencial de innovación para la política habitacional Chilena. Revista INVI, 2014. n. August, p. 79-112.

8. ___ _ FORRAY, R. La vivienda: un problema de acceso al suelo. Arq, 2014. v. 86, p. 48-57. Disponível em: <http://www.scielo.cl/pdf/arq/n86/art07.pdf >.

9. DELGADILLO, V. Mejoramiento habitacional en las áreas urbanas centrales de América Latina. del combate de tugurios a la rehabilitación habitacional progresiva. Revista INVI, 2008. v. 23, n. 63, p. 89-119.

10. DUARTE, C. G. Habitação e gestão ambiental em áreas de mananciais: o caso do município de Santo André (SP). Housing and environmental management in water source areas: the case of Santo André (SP). Saúde e Sociedade, 2012. p. 82-95.

11. FEDERAL, SENADO. Estatuto da Cidade. Guia para implementação pelos municípios e cidadãos. Brasilia, 2001.

12. FREITAS, C. S.; RENATO, L.; PEQUENO, B. Produção habitacional na região metropolitana de Fortaleza na década de 2000: avanços e retrocessos.Revista Brasileira de Estudos Urbanos e Regionais (RBEUR), 2015, v. 17, n .1, p. 45-59.

13. GONÇALVES, T. M. Habitar. A casa como contingência da condição humana. Revista INVI, 2010. p. 83-108.

14. HIDALGO, R.; ZUNINO, H. M. La urbanización de las áreas periféricas en Santiago y Valparaíso: el papel de las relaciones de poder en el dibujo de la geografía socioresidencial. Eure, 2011. v. 37, n. 111, p. 79-105. 
15. KOPPER, M. "Minha casa, minha vida”. Experts, sentidos de classe e a invenção do "mercado" da casa própria no Brasil contemporâneo. Horizontes antropológicos, 2016. v. 45, p. 185-215.

16. MACHADO, A. B. O plano diretor de Aracaju e suas contradições: uma análise preliminar. Geography department, University of Sao Paulo, 2012. v. 24, n. 2012, p. 169-184. Disponível em: <http://www.revistas.usp.br/rdg/article/view/52760>.

17. MARENGO, C. Extensión urbana e intervenciones. El caso de la ciudad de Córdoba (Argentina). Cuadernos de vivienda y urbanismo, 2013. v. 6, n. 12, p. 280-301.

18. MARSTON, S. A. The social construction of scale. Progress in human geography, 2000. v. 24, n. 2, p. 219-242.

19. MARTORI, J. C.; HOBERG, K. Población inmigrante y espacio urbano. Indicadores de segregación y pautas delocalización. Revista Eure, 2006 v. XXXII, n. 97, p. 49-62.

20. MONTEIRO, F. A. A habitação social na atualidade na região metropolitana de Curitiba/PR. Geo uerj, 2015. v. 27, p. 156-175.

21. OLIVEIRA, Júlio Cesar Pinheiro de; PEDLOWSKI, Marcos Antônio. Estado e programas municipais de habitação popular em Campos dos Goytacazes (RJ). Análise social, n. 204, p. 656-682, 2012.

22. OLIVEIRA SANTOS, E. Interfaces entre a política habitacional e o plano diretor participativo na metrópole Fortaleza-CE. Sociedade \& natureza, 2013. v. 25, n. 3, p. 485-501.

23. QUINTANA, F. Urbanizando con tiza. Arq (Santiago), 2014. n. 86, p. 30-43. Disponível em: $<$ http://www.scopus.com/inward/record.url?eid=2-s2.0-84903519311\&partnerID=tZOtx3y1>.

24. RIBEIRO, J. A. Zavaglia M. T.; MOREIRA, T. A. A política fundiária nos planos locais de habitação: entre a demanda terra e o direito de propriedade. Ambiente construído, 2014. v. 14, n. 4, p. 217- 232.

25. ROLNIK, R.; IACOVINI, R.; KLINTOWITZ, D. Habitação em municípios paulistas: construir políticas ou "rodar" programas? Estudos urbanos e regionais, 2014. v. 16, n. 2, p. 19-165.

26. RUBIN, G. O. O Problema Habitacional na América Latina: Exemplos do Brasil e Chile.Geo uerj, 2014. v. 24, p. 125-144.

27. SHIMBO, L. O aquecimento imobiliário e o mercado de habitação na área central de São Paulo (2010). Eure, 2013. v. 39, n. 117, p. 215-235.

28. VALENZUELA, I. B.; SUÁREZ, J. J. P.; DOWNEY, F. S. Vivir en campamentos: ¿camino hacia la vivienda formal o estrategia de localización para enfrentar la vulnerabilidad? Eure, 2010. v. 36, n. 109, p. 111-141.

\section{ARTIGO RECEBIDO EM OUTUBRO DE 2016}

ARTIGO APROVADO EM MARÇO DE 2017 